\title{
Giant Brain Abscess in a Neonate Managed with External Drainage
}

\section{Sedain $\mathbf{G}^{1}$, Ali ${ }^{2}$, Bhattachan $\mathbf{M}^{3}$, Sharma $\mathbf{M S}^{4}$, Shilpakar SK $^{5}$}

${ }^{1}$ Dr. Gopal Sedain, MBBS, MS, MCh, Assistant Professor, ${ }^{2}$ Dr. Niyaf Ali, MBBS, MS (MCh Resident), ${ }^{3}$ Dr. Maya Bhattachan, MBBS, MS (MCh Resident), ${ }^{4} \mathrm{Dr}$. Mohan Raj Sharma, MBBS, MS, Associate Professor, ${ }^{5} \mathrm{Dr}$. Sushil Krisna Shilpakar, Professor. All from the Department of Surgery (Neurosurgery unit), Tribhuvan University Teaching Hospital, Maharajgunj, Kathmandu, Nepal.

\section{Introduction}

B rain abscesses in neonates are rare complications of bacterial meningitis and sepsis. The most common causative organism is Gram-negative bacteria. Although brain abscess mortality is decreasing, morbidities caused by hemiparesis, seizures, visual field defects, and learning difficulties remain high. The current treatment for brain abscess involves combined medical and surgical therapy. We report a neonate with giant brain abscess which was successfully managed with aspiration and drainage with concurrent intravenous antibiotics.

\section{The Case}

A 26 day neonate was admitted with fever, irritability and poor suckling. He was full term, home delivered with birth weight of $2 \mathrm{~kg}$. At the time of presentation, he was febrile (100 degrees F), lethargic, weak cry and bulging fontanelles. He had no cranial nerve or sensori motor deficits. There was no identifiable focus of infection and no features of cyanotic heart disease. CT head revealed a large hypodense capsulated lesion suggestive of left frontal lobe abscess (Figure 1). He was operated emergently and around $80 \mathrm{ml}$ of purulent material was aspirated. An external ventricular drain (EVD) catheter was placed in the cavity and kept in place. Gram stain revealed gram negative organisms in pus however organisms could not be isolated in culture. Blood culture was sterile.

Intracavity antibiotic (Gentamycin 3mg) was given daily through the drainage catheter for a week.

\footnotetext{
Address for correspondence

Dr. Gopal Sedain, Assistant Professor

Neurosurgery Unit, Department of Surgery

Tribhuvan University Teaching Hospital, Kathmandu Nepal

E mail: gsedain@rediffmail.com
}

\begin{abstract}
Brain abscesses in neonates are extremely rare and usually occur in patients with certain risk factors. A 26 days child presented to the emergency with fever, irritability and not sucking well. CT revealed a large left frontal lobe abscess which was managed with burr hole and a drainage tube with concurrent intravenous antibiotics.
\end{abstract}

Key words: abscess, neonate, burr hole, drainage

He received empirical i/v antibiotics (Ceftriaxone, vancomycin and metrondazole). Serial CT scans were done (Figure 2,3,4) to evaluate the efficacy of treatment which revealed decreasing size of the abscess and no hydrocephalus. The EVD catheter was removed on day 12. Vancomycin and ceftriaxone were continued for 6 weeks. At 6 weeks, there was resolution of abscess and the child was playful and feeding well.

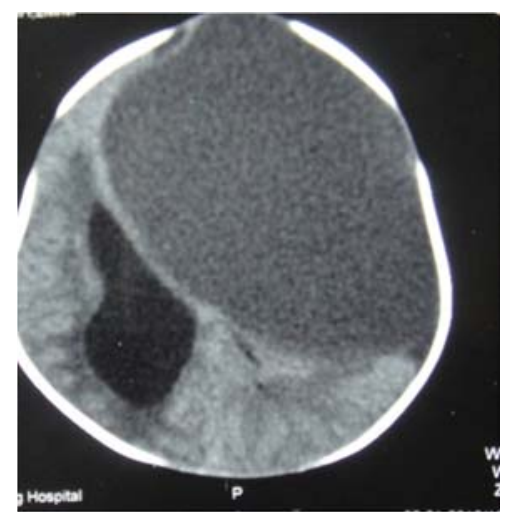

Fig 1: NCCT head at presentation showing a huge left fronto-parietal abscess. Midline shift to the right with non visualisation of left lateral ventricles can be noted.

\section{Discussion}

Common causes of brain abscess are sinusitis, otitis media, osteomyelitis and pulmonary infections. However, in infants and neonates common causes are neonatal meningitis and septicemia. In a series of 30

\section{How to cite this article?}

Sedain G, Ali N, Bhattachan M, Sharma MS, Shilpakar SK. Giant Brain Abscess in a Neonate Managed with External Drainage. J Nepal Paediatr Soc 2013;33(1):55-56. 


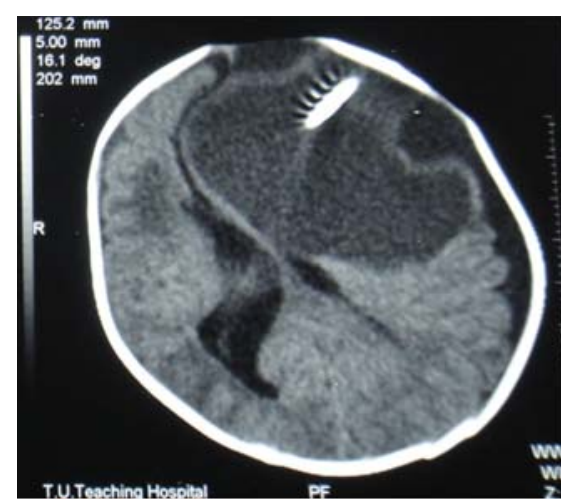

Fig 2: CT head at post op day 3 showing reduction in size of abscess. The catheter tip can be seen inside the cavity.

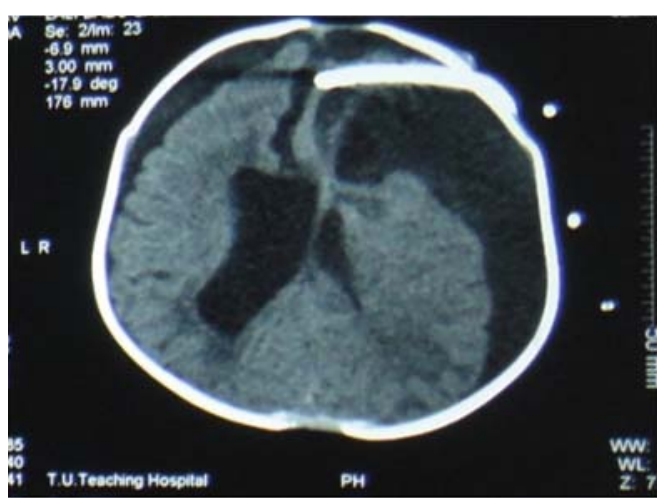

Fig 3: At 10 days post op, marked reduction in size of abscess cavity with bilateral subdural collection

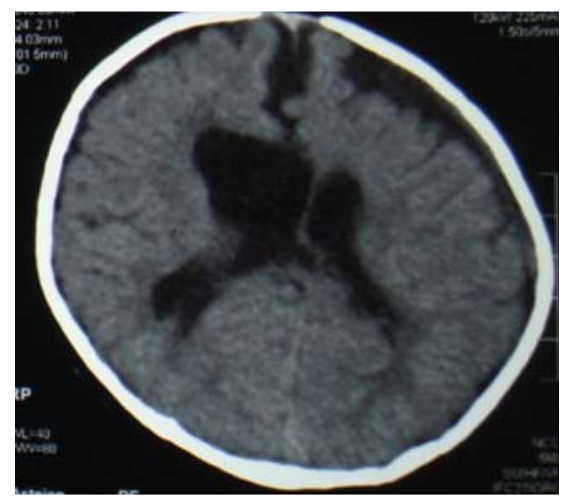

Fig 4: CT head at 6 weeks showing complete resolution of abscess

cases, Renier et al $^{1}$. reported meningitis in 20 patients and septicemia in 13. In rare cases, brain abscess can be a complication of cyanotic heart disease, compound head injury and ventriculoperitoneal shunts in children. The general presentation of patients with brain abscess includes focal deficits in 50\%-80\%, high-grade fever in $50 \%$ and signs of meningitis in $20 \%$ of cases. Common organisms in neonatal meningitis and brain abscess are reported to be Proteus mirabilis, Citrobacter and Serratia marcescens ${ }^{1,2}$. In rare cases Klebsiella pneumoniae, Enterococci faecalis, Staphylococcus aureus have also been isolated from the pus of neonatal brain abscesses $2,3,4$. Narendra et $a^{5}$ reported the optimal management of brain abscess involves surgical drainage for medium to large abscesses with eradication of the primary source. Surgical drainage was performed in $97.1 \%$ of the cases; the outcomes were good for $81.3 \%$ of them. Renier et $\mathrm{al}^{1}$. reported the following good prognostic factors in neonatal brain abscess: sterile CSF, normal ventricle on CT scan, absence of seizure and early aspiration of the abscess.

Clinical features, supported by ultrasound evaluation and CT brain scan findings, usually confirm the diagnosis and ultrasound also helps in aspiration of the brain abscess. CT scan is invaluable in diagnosis, evaluation of multiplicity and in establishing the associated pathology such as ventriculitis, hydrocephalus and infarct.

The long-term outcome of neonates with brain abscess is not very promising. Carey et $\mathrm{al}^{6}$. reported impaired scholastic ability in $70 \%$ of children tested 6 years after surgery. It also seems that the younger the child at the time of diagnosis, the worse is the prognosis for intellectual development. Renier et $\mathrm{al}^{1}$ performed IQ testing in 22 children-among them eight had an IQ above 80 and another eight patients had an IQ less than 60 . Thus there is a very high risk of mental subnormality in these children.

\section{Conclusion}

Brain abscess in neonate is relatively uncommon but has high mortality and morbidity. Early intervention and management can yield good results.

\section{References}

1. Renier D, Flandin C, Hirch E, Hirch JF. Brain abscess in neonate. A study of 30 patients. J Neurosurg 1988;69:877-82.

2. Pant P, Banerjee S, Ganguly S. Klebsiella pneumoniae brain abscess in two neonates. Indian Pediatr 2008;45(8):693-694.

3. Mahapatra AK, Pawar SJ, Sharma RR. Brain abscess due to Staphylococcus aureus following neonatal breast abscess: case report and a brief review of the literature. Ann Saudi Med 2001;21:8083.

4. Sundaram V, Agrawal S, Chacham S, Mukhopadhyay K, Dutta S, Kumar P. Klebsiella pneumoniae brain abscess in neonates: a report of 2 cases. J Child Neurol 2010;25(3):379-82.

5. Nathoo N, Nadvi SS, Narotam PK, van Dellen JR. Brain abscess: management and outcome analysis of a computed tomography era experience with 973 patients. World Neusurg 2011;75:716-266.

6. Carey ME, Chou SN, French LA. Experience with brain abscess. J Neurosurg 1972;36:1-9. 